\title{
THz BACKWARD-WAVE OSCILLATORS FOR PLASMA DIAGNOSTIC IN NUCLEAR FUSION
}

\author{
Claudio Paoloni ${ }^{1}$, Lingna Yue ${ }^{3}$, Xiaopin Tang ${ }^{3}$, Fuzhi Zhang ${ }^{4}$, \\ Branko Popovic ${ }^{2}$, Logan Himes ${ }^{2}$, Robert Barchfeld ${ }^{2}$, \\ Diana Gamzina ${ }^{2}$, Rosa Letizia ${ }^{1}$, Mauro Mineo ${ }^{1}$, and \\ Neville C. Luhmann Jr. ${ }^{2}$ \\ ${ }^{1}$ Lancaster University, Lancaster ,UK \\ ${ }^{2}$ University of California Davis, Davis, USA \\ ${ }^{3}$ University of Electronic Science and Technology of China, \\ Chengdu, China \\ ${ }^{4}$ Beijing Vacuum Electronics Research Institute, China
}

The understanding of plasma turbulence in nuclear fusion is related to the availability of powerful $\mathrm{THz}$ sources and the possibility to map wider plasma regions. A novel approach to realize compact $\mathrm{THz}$ sources to be implemented in the plasma diagnostic at NSTX experiment (Princeton Plasma Physics Laboratory, USA) is reported.

Two novel $0.346 \mathrm{THz}$ Backward-Wave Oscillators (BWOs) have been designed and are presently in the fabrication phase. One BWO is based on the Double Staggered Grating ${ }^{1}$ (DSG) that supports a sheet electron beam to provide a high output power; the second BWO is based on the Double Corrugated Waveguide $^{2}$ (DCW) that supports a cylindrical electron beam generated by a conventional Pierce gun.

The performance of both the BWOs was computed by Particle-in-cells (PIC) simulations. The DSG-BWO provides about $1 \mathrm{~W}$ of output power with a beam current of $10 \mathrm{~mA}$ and a beam voltage of $16.8 \mathrm{kV}$.

The DCW-BWO provides $0.74 \mathrm{~W}$ output power with $10 \mathrm{~mA}$ beam current and $13 \mathrm{kV}$ beam voltage.

The DSG and the DCW have been realized by state of the art prototype nano-CNC milling machine (DMG Mori-Seiki) that permits one to achieve performance, in term of cost and surface finishing, unavailable with any other technology. It is the first time that this technique is applied to structures above $0.3 \mathrm{THz}$.

The high output power of both the BWOs demonstrates the importance of novel approaches in the emerging field of $\mathrm{THz}$ vacuum electron devices.

1. Y.-M. Shin, L. R. Barnett, and N. C. Luhmann, "Strongly confined plasmonic wave propagation through an ultrawideband staggered double grating waveguide," Applied Physics Letters, vol. 93, no. 22, p. 221504, 2008

2. M. Mineo and C. Paoloni, "Double-Corrugated Rectangular Waveguide Slow-Wave Structure for Terahertz Vacuum Devices," IEEE Trans. Electron Devices, vol. 57, no. 11, pp. 3169-3175, Nov. 2010.

This work is supported by UK EPSRC EP/L026597/1 grant and US NSF CHE-1429258 grant 